\title{
Euglycemic diabetic ketoacidosis associated with uremia
}

\author{
Mingxia Zhang ${ }^{1}$, Haiyan Xiang ${ }^{1}$, Hui Fan ${ }^{1}$, and Lunzhi Liu ${ }^{1}$ \\ ${ }^{1}$ Minda Hospital of Hubei Minzu University
}

July 7,2020

\begin{abstract}
Regular treatment of DKA patients includes rapid and large amount of fluids supply, which is not feasible in DKA patients associated with Uremia because of being urineless and prone to heart failure of these patinets. Our results indicated that continuous renal replacement therapy (CRRT) is a better alternative.
\end{abstract}

\section{INTRODUCTION}

As an acute complication of diabetes, diabetic ketoacidosis (DKA) is typically characterized with hyperglycemia $\left(16.7-33.3 \mathrm{mmol} / \mathrm{L},>55.5 \mathrm{mmol} / \mathrm{L}\right.$ in rare case), elevated serum ketones, and acidosis ${ }^{1}$. Whereas DKA patients with normal blood glucose concentrations $(<16.7 \mathrm{mmol} / \mathrm{L})$ and low bicarbonate levels $(<10$ $\mathrm{mmol} / \mathrm{L}$ ) were also reported, the pioneer of which is Munro et al. in $1973{ }^{2}$.

The optimal emergence treatment of DKA is fluids supply, which can increase blood volume and restore efficient influx of organs. However, in regarding to patients at the last stage of diabetic nephropathy relying on sustainable hemodialysis, rapid and large amount of fluids supply is not feasible since they have no urine and can easily get heart failure. In this group of patients, normal fluids/blood supply can only correct the acidosis for short time. Ketones in tissues will enter the blood and cause acidosis in two to three hours.

For these cases, continuous renal replacement therapy (CRRT) is a better option in many ways, including large amount fluids exchange, increased influx of organs, clearance of keto acid, lactic acid and inflammatory mediator in tissues, enhanced efficiency of essential molecules in blood, improved microcirculation, remission of tissue hypoxia. Up to now, there is no report of the treatment of DKA associated with uremia.

Our case report here indicate that normal dialysis could not correct DKA associated with uremia, while CRRT is the best available option for treatment.

\section{Key Words}

Uremia, Euglycemia, DKA

\section{Key Clinical Message}

Regular treatment of DKA patients is not feasible in cases associated with Uremia because of being urineless and prone to heart failure of these patinets. Our results indicated that continuous renal replacement therapy is a better alternative.

\section{CASE REPORT}

We describe a case of a 26-yr-old female with type I diabetes (T1DM) of more than 10-yr duration. This patient presented with severe abdominal pain started one day ago and had been performing continuous ambulatory peritoneal dialysis for one year. This patient had a history of tuberculosis which was cured already. Ten years ago when this patient was diagnosed with T1DM after showing polydipsia without 
obvious trigger, she was treated with insulin injection. One year ago this patient was hospitalized for uremia (late stage of chronic renal failure). Peritoneal dialysis catheter placement was performed and followed with regular peritoneal dialysis, after which this patient's clinical evolution was favorable.

The patient was hospitalized because of a 24 -hr history of durative blunt abdominal pain. The associated symptoms included mushy stool diarrhea, cloudy peritoneal dialysate fluid. The patient had no sign of dark stool, chills or fever. On physical examination the patient showed signs of moderate anemia-like paleness. The patient was alert, with vital signs in normal range which include temperature at 36.5, pulse rate at 80 $\mathrm{bpm}$, respiratory rate at $20 \mathrm{bpm}$, blood pressure at 100/60 $\mathrm{mmHg}$. The physical examination showed no jaundice-yellow with skin, sclera and mucous membranes, no swelling of superficial lymph nodes. The breath sounds are clear without obvious adventitious sounds like dry/moist rales. Abdominal swelling caused by accumulation of fluid was observed. There was no observation of hepatosplenomegaly, tenderness or rebound tenderness, as well as peripheral edema. The patient showed normal reflex action and no sign of pathological spread of reflexes.

The blood test results when hospitalized were as follows, leukocytes $11.8 \times 10^{9} / \mathrm{L}$, hemoglobin $65 \mathrm{~g} / \mathrm{L}$, Creactive protein $(\mathrm{CRP}) 140.94 \mathrm{mg} / \mathrm{L}$, albumin $19.1 \mathrm{~g} / \mathrm{L}$, creatinine $528.0 \mu \mathrm{mol} / \mathrm{L}$, blood glucose $15.0 \mathrm{mmol} / \mathrm{L}$ and potassium $2.58 \mathrm{mmol} / \mathrm{L}$. The peritoneal fluid was cloudy, and the analysis of which showed leukocytes at $80 \%$ and nucleated cell count at $2.93 \times 10^{9} / \mathrm{L}$. The suspected bacterial infection was treated with intravenous and peritoneal administration of cefazolin and levofloxacin lactate upon hospitalization. Measures were taken to adjust the levels of blood glucose and blood pressure, correct anemia, maintain the level of electrolytes and $\mathrm{pH}$ balance. On Oct $18^{\text {th }}$, the drug resistance result from culture of peritoneal dialysis fluid indicated the infection of Candida dubliniensis. Treatment was then switched to intravenous Fluconazole and intraperitoneal influx of antifungal medication. At the meantime, peritoneal dialysis catheters were removed and blood dialysis was performed. On Oct $25^{\text {th }}$, the abdominal pain got worse and the patient showed obvious tenderness and rebound tenderness, which indicated intestinal obstruction. The intake of water and food was forbidden and gastrointestinal decompression was performed. In addition to the antifungal medication, Piperacillin/Tazobactam was prescribed against the infection. At $12 \mathrm{am}$ of Oct $29^{\text {th }}$, the patient felt chest tightness and shortness of breath. The blood pressure was taken at $80 / 50 \mathrm{mmHg}$. The patient was in extremely low spirits. Obvious moist rales was noticed. Test of blood dialysis from Oct $27^{\text {th }}$ read $\mathrm{BNP}>10000, \mathrm{CO}_{2} \mathrm{CP} 6.4 \mathrm{mmol} / \mathrm{L}$, blood glucose $15 \mathrm{mmol} / \mathrm{L}$. Beside the supply of fluid, dopamine was given to correct the risk of shock. Dialysis was emergency performed against acidosis. The CRP level was measured again at $175 \mathrm{mg} / \mathrm{L}$, calcitonin at 4.48 , leukocytes count at $16.67 \times 10^{9} / \mathrm{L}$. Again the abdominal pain got worse and the anti-infection reagent was switched to Meropenem. At the noon, $\mathrm{CO}_{2} \mathrm{CP}$ was read at $6.4 \mathrm{mmol} / \mathrm{L}, \mathrm{pH}$ at 7.17 . Dialysis was performed in the afternoon and again on Oct $30^{\text {th }}$. On Oct $31^{\text {st }}$, $\mathrm{CO}_{2} \mathrm{CP}$ was read at $9.7 \mathrm{mmol} / \mathrm{L}$, blood glucose at $15 \mathrm{mmol} / \mathrm{L}$ (hypoglycemia). Multiple blood gas tests indicated metabolic acidosis whereas the level of lactic was normal. The blood glucose level was increased although not as significant as seen in DKA. No urine was available to measure the level of ketones and it is possible that urine ketones could be negative in DKA associated with chronic kidney disease (CKD). Other local hospitals did not have the ability to test the blood ketones either. So the dialysis fluid was used as urine to test for ketones, which showed positive. This patient was then diagnosed as ketoacidosis and CRRT was performed accordingly. The acidosis was following corrected and the blood sample was confirmed with ketoacidosis after sending out for test.

\section{DISSCUSSION}

Previous study indicate that DKA can be precipitated by infection ${ }^{1,3}$. In our case, the patient was confirmed with infection of Candida dubliniensis. At the meanwhile, the patient also showed signs of intestinal obstruction, so we took measures of fasting, which could be another cause for DKA ${ }^{3,4}$. Our case also indicated that in patients of DKA associated with uremia, ketones analysis can be performed using the dialysis fluid in case of no urine available. Most importantly, our report suggested that for DKA associated with uremia patients, hemodialysis was not able to correct acidosis. In our case, management strategies for DKA should be modified, which is consistent with previous findings that current management strategies for DKA 
is not suitable to patients with end-stage renal disease (ESRD) ${ }^{5}$. Moreover, our example provided the evidence that CRRT can be an optimal strategy to treat DKA associated with uremia, which can serve as an alternative approach for DKA patients associated with ESRD.

Conflict of interest: The authors declare no conflicts of interest in association with the present study.

Data availability statement: The data that support the findings of this study are available from the corresponding author upon reasonable request.Author contribution: MZ and LL analyzed the data and wrote the paper. HX and HF assisted with the data collection and analyze. All authors read and approved the final manuscript.

\section{REFERENCES}

1. Thawabi, M. \& Studyvin, S. Euglycemic Diabetic Ketoacidosis, a Misleading Presentation of Diabetic Ketoacidosis. N. Am. J. Med. Sci. 7 , 291-4 (2015).

2. Munro, J. F., Campbell, I. W., McCuish, A. C. \& Duncan, L. J. P. Euglycaemic Diabetic Ketoacidosis. BMJ 2 , 578-580 (1973).

3. Dowsett, J., Humphreys, R. \& Krones, R. Normal Blood Glucose and High Blood Ketones in a Critically Unwell Patient with T1DM Post-Bariatric Surgery: a Case of Euglycemic Diabetic Ketoacidosis. Obes. Surg. 29 , 347-349 (2019).

4. Baena, M. G., Cayón, M., Ortego-Rojo, J. \& Aguilar-Diosdado, M. Diabetic ketoacidosis associated with severe hypoglycemia. J. Endocrinol. Invest. 33 , 358-359 (2010).

5. Schaapveld-Davis, C. M. et al. End-Stage Renal Disease Increases Rates of Adverse Glucose Events When Treating Diabetic Ketoacidosis or Hyperosmolar Hyperglycemic State. Clin. Diabetes35 , 202-208 (2017). 